\title{
Well-posedness of a Neumann-type problem on a gauge ball in $\mathrm{H}$-type groups
}

\author{
Mukund Madhav Mishra ${ }^{1}$ and Ashutosh Pandey ${ }^{2 *}$
}

\section{"Correspondence:}

ashutoshpandey4521@gmail.com

2Department of Mathematics,

Faculty of Mathematical Sciences,

University of Delhi, Delhi, India

Full list of author information is

available at the end of the article

\section{Springer}

\begin{abstract}
We discuss the existence and uniqueness of solution for the second boundary value problem of potential theory often referred to as the Neumann problem, on a gauge ball for the canonical sub-Laplacian in H-type groups. In this way we extend the classical results of the problem as well as its generalization to the Heisenberg group.
\end{abstract}

MSC: 31B20; 35H20; 35N15; 45B05

Keywords: Neumann problem; H-type groups; Sub-Laplacian; Horizontal normal vectors

\section{Introduction}

In the study of partial differential equations, two boundary value problems associated with the Laplace equation occupy a special place, namely the Dirichlet and Neumann problems. While the Dirichlet problem asks to obtain a harmonic function in a domain whose value agrees with a prescribed (continuous) function on the boundary, the Neumann problem requires the normal derivative of the solution function to agree with a prescribed function [17]. If $\Delta$ denotes the Laplacian on the Euclidean space $\mathbb{R}^{n}$, the Neumann problem for a smooth domain $\Omega$ in $\mathbb{R}^{n}$ is finding a $u \in C^{2}(\Omega) \cap C^{1}(\bar{\Omega})$ such that

$$
\begin{cases}\Delta u=0 & \text { in } \Omega, \\ \frac{\partial u}{\partial n}=f & \text { on } \partial \Omega,\end{cases}
$$

where $f$ is a prescribed function on $\partial \Omega$ and $n$ is the outward normal at the boundary $\partial \Omega$. For domains having $C^{1, \alpha}$ boundary where $\alpha$ is in $(0,1]$, for example, a unit ball, the Dirichlet problem is solvable for any continuous boundary value (see, for details, [10]) whereas the Neumann problem is solvable under the essential condition that the integral of the values assigned to the normal derivative vanishes over the boundary surface (see, for details, [14]), i.e.,

$$
\int_{\partial \Omega} f d s=0
$$

(c) The Author(s) 2020. This article is licensed under a Creative Commons Attribution 4.0 International License, which permits use, sharing, adaptation, distribution and reproduction in any medium or format, as long as you give appropriate credit to the original author(s) and the source, provide a link to the Creative Commons licence, and indicate if changes were made. The images or other third party material in this article are included in the article's Creative Commons licence, unless indicated otherwise in a credit line to the material. If material is not included in the article's Creative Commons licence and your intended use is not permitted by statutory regulation or exceeds the permitted use, you will need to obtain permission directly from the copyright holder. To view a copy of this licence, visit http://creativecommons.org/licenses/by/4.0/. 
A straight generalization of these boundary value problems involving the Laplacian in the case of $\mathbb{R}^{n}$ by replacing the operator $\Delta$ with an arbitrary elliptic operator has been vastly discussed, and an extensive literature is available $[7,10,14,18,19]$. These problems become more interesting when the regularity of the differential operator involved is compromised. The conditions of being hypoelliptic and subelliptic, that are well exposed in the classical book [4], are weaker than ellipticity. In some sense, the first and nicest example of a subelliptic operator is the Kohn-Laplacian on the Heisenberg group. The discussions of boundary value problems involving the sub-Laplacian sometimes are parallel to the classical cases, while are considerably different in certain other cases. The Dirichlet problem for the Kohn-Laplacian on the Heisenberg groups was first discussed by Gaveau [9] and then settled completely by Jerison $[11,12]$. The Neumann boundary value problem on the Heisenberg group has been recently discussed by Dubey et al. [6]. The Heisenberg group case, however, comes with an explicit group law and the underlying manifold structure same as that of the Euclidean space $\mathbb{R}^{2 n+1}$. Therefore, the case of Heisenberg group in [6], up to a certain extent, is same as dealing with a subelliptic operator on Euclidean spaces. The point where it shows contrast with the Euclidean setup is the Green's-type identity where, instead of the Lebesgue measure, one needs a different volume and surface element in the integrals.

The immediate generalization, namely the H-type groups, is by definition the range of the exponential map on an $\mathrm{H}$-type Lie algebra. The group law on an H-type group, therefore, is not as explicit as in the case of Heisenberg group. Moreover, the manifold structure, which is still trivial, does not enjoy a natural coordinate system and there arises a need to find one that is most compatible with the Lie algebra structure. It is natural to see the extent to which the techniques can be generalized and equally natural to start with the H-type groups. The identification of characteristic points on the boundary and estimation of integrals involved are two focal areas in the work of Dubey et al. [6]. The case of $\mathrm{H}$-type groups in this article has established that similar results are available in this setup and hence opens the scope to study Neumann boundary value problems for subelliptic operators on a more general class of 2-step nilpotent Lie groups [2,3].

The plan of our article is as follows. In Sect. 2, we refer to $[3,5]$ and review some basic aspects of the H-type groups, sub-Laplacian, and the horizontal normal vectors. Analogous to the normal derivative, we define a similar operator in Sect. 3 to deal with the characteristic points and formulate the Neumann boundary value problem. In the subsequent sections, we prove a few results as a build-up to the main result in this article.

\section{H-type group and horizontal normal vectors}

We first recall the definition of Heisenberg-type group (or H-type group) as a 2-step nilpotent Lie group whose Lie algebra admits certain properties.

Let $\mathbf{g}$ be a real Lie algebra equipped with an inner product such that it can be represented as an orthogonal direct sum

$$
\mathbf{g}=\mathfrak{v} \oplus \mathfrak{z}
$$

where $\mathfrak{z}$ is the center of $\mathbf{g},[\mathfrak{v}, \mathfrak{v}] \subseteq \mathfrak{z}$ and $[\mathfrak{v}, \mathfrak{z}]=[\mathfrak{z}, \mathfrak{z}]=\{0\}$. Define a linear mapping $J: \mathfrak{z} \rightarrow$ $\operatorname{End}(\mathfrak{v})$ as

$$
\left\langle J_{Z} X, X^{\prime}\right\rangle=\left\langle Z,\left[X, X^{\prime}\right]\right\rangle \quad \forall X, X^{\prime} \in \mathfrak{v} \text { and } Z \in \mathfrak{z} .
$$


Then $\mathbf{g}$ is called an H-type Lie algebra if for all $Z \in \mathfrak{z}$,

$$
J_{Z}^{2}=-|Z|^{2} I
$$

where $I$ is the identity mapping. A connected and simply connected Lie group $\mathbf{G}$ with $\mathbf{g}$ as the associated Lie algebra is called an $\mathrm{H}$-type group. The Heisenberg group $\mathbb{H}_{n}$ is a trivial example of an $\mathrm{H}$-type group and a nontrivial example will be discussed in the next section.

We identify $\mathbf{g}$ with the corresponding simply connected Lie group $\mathbf{G}$ under the exponential map. From [16], the product in $\mathbf{G}$ is given by

$$
(X, Z)\left(X^{\prime}, Z^{\prime}\right)=\left(X+X^{\prime}, Z+Z^{\prime}+\frac{1}{2}\left[X, X^{\prime}\right]\right)
$$

Denote by $p$ and $q$ the dimensions of $\mathfrak{v}$ and $\mathfrak{z}$, respectively, so that $p+2 q$ is the homogeneous dimension of $\mathbf{G}$. When $X$ is regarded as a left-invariant vector field on $\mathbf{G}$ at the point $\left(X^{\prime}, Z^{\prime}\right)$, it can be represented as

$$
X=D_{X}+\frac{1}{2} D_{\left[X, X^{\prime}\right]}
$$

We fix $\left\{X_{1}, X_{2}, \ldots, X_{p}\right\}$ and $\left\{Z_{1}, Z_{2}, \ldots, Z_{q}\right\}$ as orthonormal bases for $\mathfrak{v}$ and $\mathfrak{z}$, respectively, so that $\left\{X_{1}, X_{2}, \ldots X_{p}, Z_{1}, Z_{2}, \ldots, Z_{q}\right\}$ is an orthonormal basis of $\mathbf{g}$. Then $X=\sum_{j=1}^{p}\left\langle X, X_{j}\right\rangle X_{j}$ and $Z=\sum_{j=1}^{q}\left\langle Z, Z_{j}\right\rangle Z_{j}$ for every $X \in \mathfrak{v}$ and $Z \in \mathfrak{z}$.

The canonical sub-Laplacian on $\mathbf{G}$ is given by

$$
\Delta_{\mathbf{G}}=\sum_{j=1}^{p} X_{j}{ }^{2} .
$$

Define $\mathbf{p}(\alpha)=\left(\frac{|X|^{4}}{16}+|Z|^{2}\right)^{\frac{1}{4}}$ as the gauge of an element $\alpha=(X, Z) \in \mathbf{G}$.

Theorem 2.1 (Kaplan [13]) There exists a positive constant c such that

$$
g(\alpha):=c\left({\frac{|X|^{4}}{16}}^{4}+|Z|^{2}\right)^{-(p+2 q-2) / 4}
$$

is the fundamental solution of the sub-Laplacian, that is,

$$
\Delta_{\mathbf{G}} g_{\beta}=-\delta_{\beta},
$$

where $g_{\beta}(\alpha)=g\left(\beta^{-1} \alpha\right)$ and $\delta_{\beta}$ is the Dirac distribution with pole at $\beta \in \mathbf{G}$.

Just for the sake of calculations, we use a slightly modified kernel $\Phi$ where

$$
\Phi(\alpha, \beta)=2 g_{\beta}(\alpha)
$$

Unless stated otherwise, all the derivatives of $\Phi$ would be taken with respect to the first variable $\alpha$. 
An infinitesimal metric that is consistent with the automorphisms of an H-type group happens to be a sub-Riemannian metric obtained by Korányi in [15]. We define

$$
\left\langle Y, Y^{\prime}\right\rangle_{0}=-4 b^{2} B\left(Y, \theta Y^{\prime}\right)
$$

for all $Y, Y^{\prime} \in \mathbf{g}$ where $B$ denotes the killing form, $\theta$ is the Cartan involution, and $4 b^{2}=$ $(p+4 q)^{-1}$.

Definition 2.2 A vector at any point $\alpha \in \mathbf{G}$ is called horizontal if it is of the form $X_{\alpha}$ ( $X \in$ $\mathfrak{v})$. The length of a horizontal vector is given by $\left\|X_{\alpha}\right\|_{0}=|X|$ where $|\cdot|$ denotes the norm induced by $\langle\cdot, \cdot\rangle$ on $\mathfrak{v}$.

Vectors that are not horizontal are said to have infinite length. We call the unique horizontal vector $\nabla_{0} f$, the horizontal gradient of a function $f$ on $\mathbf{G}$ which is defined as $\left\langle\nabla_{0} f, w\right\rangle_{0}=w \cdot f$ for all horizontal vectors $w$. We also have

$$
\nabla_{0} f=\sum_{j=1}^{p}\left(X_{j} f\right) X_{j}
$$

where $\left\{X_{j}\right\}_{j=1}^{p}$ is an orthonormal basis in $\mathfrak{v}$.

The outward horizontal normal unit vector at each point of the boundary of a domain $\{F<0\}$ where $\left\|\nabla_{0} F\right\|_{0} \neq 0$ is given by

$$
\frac{\partial}{\partial n_{0}}=\frac{1}{\left\|\nabla_{0} F\right\|_{0}} \nabla_{0} F
$$

\section{Formulation of the problem}

The unit horizontal normal vector in Eq. (2.5) is undefined at the "characteristic" points, i.e., the points where $\nabla_{0} F$ vanishes. When $F$ is smooth, the set of characteristic points forms a lower-dimensional subset of the boundary. In the following example, we explicitly calculate the set of characteristic points on an H-type group.

Example 3.1 Consider the group

$$
\mathbf{G}=\mathbb{R}^{4} \oplus \mathbb{R}^{2}
$$

with the following binary operation:

$$
(x, z) \circ(y, \omega)=\left(x+y, z_{1}+\omega_{1}+\frac{1}{2}\left\langle T_{1}(x), y\right\rangle, z_{2}+\omega_{2}+\left\langle T_{2}(x), y\right\rangle\right),
$$

where $x=\left(x_{1}, x_{2}, x_{3}, x_{4}\right) \in \mathbb{R}^{4}, z=\left(z_{1}, z_{2}\right) \in \mathbb{R}^{2}$,

$$
T_{1}=\frac{1}{\sqrt{2}}\left[\begin{array}{cccc}
0 & 1 & 1 & 0 \\
-1 & 0 & 0 & -1 \\
-1 & 0 & 0 & 1 \\
0 & 1 & -1 & 0
\end{array}\right] \text { and } T_{2}=\frac{1}{\sqrt{2}}\left[\begin{array}{cccc}
0 & -1 & 1 & 0 \\
1 & 0 & 0 & -1 \\
-1 & 0 & 0 & -1 \\
0 & 1 & 1 & 0
\end{array}\right] \text {. }
$$


Here, $\mathbb{R}^{2}$ serves as the center of $\mathbf{G}$. We consider the Euclidean inner product on $\mathbf{G}$. The left-invariant vector field $X_{j}$ on $\mathbf{G}$ that equals $\frac{\partial}{\partial x_{j}}$ at the origin is given by

$$
X_{j}=\frac{\partial}{\partial x_{j}}+\frac{1}{2} \sum_{k=1,2}\left(\sum_{i=1}^{4} t_{(j, i)}^{k} x_{i}\right) \frac{\partial}{\partial z_{k}},
$$

where $t^{k}(j, i)$ is the $(j, i)$ th entry in $T_{k}$.

The left-invariant vector field $Z_{j}$ on $\mathbf{G}$ that equals $\frac{\partial}{\partial z_{j}}$ at the origin is given by $Z_{j}=\frac{\partial}{\partial z_{j}}$. Denote by $\mathbf{g}$ the associated Lie algebra of $\mathbf{G}$ and represent it as

$$
\mathbf{g}=\mathfrak{v} \oplus \mathfrak{z}
$$

We also denote $\left\{X_{j}\right\}_{j=1}^{4}$ and $\left\{Z_{k}\right\}_{k=1,2}$ as the orthonormal bases for $\mathfrak{v}$ and $\mathfrak{z}$, respectively. Clearly, $[\mathbf{g}, \mathfrak{z}]=\{0\}$ and $[\mathbf{g}, \mathbf{g}] \subseteq \mathfrak{z}$. For any $X \in \mathfrak{v}$ and $Z \in \mathfrak{z}$, we define a map $J_{Z}: \mathfrak{v} \rightarrow \mathfrak{v}$ as

$$
\begin{aligned}
J_{Z} X= & \frac{1}{\sqrt{2}}\left\{\left\{b_{1}\left(a_{2}+a_{3}\right)+b_{2}\left(-a_{2}+a_{3}\right)\right\} X_{1}+\left\{b_{1}\left(-a_{1}-a_{4}\right)+b_{2}\left(a_{1}-a_{4}\right)\right\} X_{2}\right. \\
& \left.+\left\{b_{1}\left(-a_{1}+a_{4}\right)+b_{2}\left(-a_{1}-a_{4}\right)\right\} X_{3}+\left\{b_{1}\left(a_{2}-a_{3}\right)+b_{2}\left(a_{2}+a_{3}\right)\right\} X_{4}\right\},
\end{aligned}
$$

where $X=a_{1} X_{1}+a_{2} X_{2}+a_{3} X_{3}+a_{4} X_{4}, Z=b_{1} Z_{1}+b_{2} Z_{2}$, and each $a_{i}, b_{j}$ is a smooth function on $\mathbf{G}$. As $J_{Z}{ }^{2}=-|Z|^{2} I$, we finally conclude that $\mathbf{G}$ is an $\mathrm{H}$-type group.

Now, let $F(X, Z)=\frac{|X|^{4}}{16}+|Z|^{2}-1=0$ be a smooth surface in G. Substituting $\nabla_{0} F=0$, we obtain

$$
\begin{aligned}
& X_{1} F=\frac{1}{2} x_{1}|x|^{2}+z_{1}\left(x_{2}+x_{3}\right)+z_{2}\left(-x_{2}+x_{3}\right)=0, \\
& X_{2} F=\frac{1}{2} x_{2}|x|^{2}+z_{1}\left(-x_{1}-x_{4}\right)+z_{2}\left(x_{1}-x_{4}\right)=0, \\
& X_{3} F=\frac{1}{2} x_{3}|x|^{2}+z_{1}\left(-x_{1}+x_{4}\right)+z_{2}\left(-x_{1}-x_{4}\right)=0, \\
& X_{4} F=\frac{1}{2} x_{4}|x|^{2}+z_{1}\left(x_{2}-x_{3}\right)+z_{2}\left(x_{2}+x_{3}\right)=0,
\end{aligned}
$$

which further gives $X=0$. Hence, the set of characteristic points is given by $\left\{(0, Z):|Z|^{2}-\right.$ $1=0\}$ which is a sphere of dimension $(q-1)$. Thus, the set of characteristic points is a lower-dimensional subset of the boundary, which finishes the example.

Now we formulate the Neumann problem. Let $\Omega$ be a bounded domain in $\mathbf{G}$ whose boundary is given as a level set of a smooth function $T$, i.e., $\partial \Omega=\{\gamma \in \mathbf{G}: T(\gamma)=0\}$. To deal with the set of characteristic points in $\mathbf{G}$, we define a new class of functions

$$
\begin{aligned}
\mathfrak{C}(\Omega)= & \left\{f \in C^{2}(\Omega) \cap C(\bar{\Omega}): \lim _{x \rightarrow x_{0}} \frac{\partial}{\partial n_{0}} f(x)\right. \text { exists for all characteristic points } \\
& \left.x_{0} \in \partial \Omega\right\},
\end{aligned}
$$


where the limit is taken with respect to the relative topology of $\bar{\Omega}$. Define the operator $\partial^{\perp}: \mathfrak{C}(\Omega) \rightarrow C(\partial \Omega)$ as

$$
\partial^{\perp} f\left(\gamma_{0}\right)= \begin{cases}\lim _{\gamma \rightarrow \gamma_{0}} \frac{\partial f}{\partial n_{0}}(\gamma) & \text { if } \gamma_{0} \text { is a characteristic point on } \partial \Omega \\ \frac{\partial f}{\partial n_{0}}\left(\gamma_{0}\right) & \text { if } \gamma_{0} \text { is non-characteristic point on } \partial \Omega\end{cases}
$$

As the set of characteristic points is a lower-dimensional subset of $\partial \Omega$, the Green's formula [16, Eq. (1.9)] can be rewritten as

$$
\int_{\Omega}\left(u \Delta_{\mathbf{G}} v-v \Delta_{\mathbf{G}} u\right) d \mu=\int_{\partial \Omega}\left(u \partial^{\perp} v-v \partial^{\perp} u\right) d S,
$$

where

$$
d S=\frac{\left\|\nabla_{0} T\right\|_{0}}{\|\nabla T\|} d s
$$

and $d s$ is the surface element on $\partial \Omega$ determined by the Euclidean measure. The homogeneous Neumann problem for a domain $\Omega$ in $\mathbf{G}$ is to find a function $u \in \mathfrak{C}(\Omega)$ such that

$$
\begin{cases}\Delta_{\mathbf{G}} u=0 & \text { in } \Omega, \\ \partial^{\perp} u=g & \text { on } \partial \Omega\end{cases}
$$

where $g \in C(\partial \Omega)$.

\section{Uniqueness}

Definition 4.1 Given $\phi \in C(\partial \Omega)$, for $\beta \in \mathbf{G} \backslash \partial \Omega$, define

$$
V(\beta):=\int_{\partial \Omega} \phi(\alpha) \Phi(\alpha, \beta) d S(\alpha) \quad \text { and } \quad \tilde{V}(\beta):=\int_{\partial \Omega} \phi(\alpha) \partial^{\perp} \Phi(\alpha, \beta) d S(\alpha) .
$$

Both $V$ and $\tilde{V}$ are $\Delta_{\mathbf{G}}$-harmonic and are respectively called the single- and the doublelayer potentials with density $\phi$.

Lemma 4.2 (Green's first identity) Let $\Omega$ be any bounded domain in $\mathbf{G}$ having boundary of class $C^{\infty}$ and $u, v$ be $C^{1}$ functions on $\bar{\Omega}$, then

$$
\int_{\partial \Omega} v \partial^{\perp} u d S=\int_{\Omega}\left(v \Delta_{\mathbf{G}} u+\nabla_{0} v \cdot \nabla_{0} u\right) d \mu .
$$

Proof The Divergence theorem [8, Corollary 3.11] when applied to $v \nabla_{0} u$ proves the above lemma.

Lemma 4.3 If $\nabla_{0} u$ is zero on every open subset $U$ of a connected domain $\Omega$ then $u$ is constant on $\Omega$.

Proof The proof follows along similar lines as that of [6, Theorem 3.2]

Theorem 4.4 Let $\Omega$ be a bounded domain with smooth boundary, then the solution of (3.2), if it exists, is unique up to an additive constant. 
Proof The difference $u=u_{1}-u_{2}$ of two solutions of the Neumann problem is a harmonic function in $\Omega$, continuous up to boundary, satisfying the homogeneous boundary condition $\partial^{\perp} u=0$ on $\partial \Omega$. Using Lemma 4.2, we get

$$
\int_{\Omega}\left|\nabla_{0} u\right|^{2} d \mu=\int_{\partial \Omega} u \partial^{\perp} u d S-\int_{\Omega} u\left(\Delta_{\mathbf{G}} u\right) d \mu=0 .
$$

Thus, $\nabla_{0} u=0$. Further using Lemma 4.3, $u$ must be a constant.

In the rest of the article, $\Omega$ will denote the open unit ball $\{\alpha=(X, Z) \in \mathbf{G}: \mathbf{p}(\alpha)<1\}$ in G.

Lemma 4.5 Let $\partial \Omega$ be of class $C^{2}$ and $\phi \in C(\partial \Omega)$. For $\beta \in \partial \Omega$, the integral $V(\beta)=$ $\int_{\partial \Omega} \phi(\alpha) \Phi(\alpha, \beta) d S(\alpha)$ exists and $V$ is continuous throughout $\mathbf{G}$.

Proof From Theorem 2.1 and Eq. (2.3), we have

$$
|\Phi(\alpha, \beta)|=2 c \mathbf{p}\left(\beta^{-1} \alpha\right)^{-(p+2 q-2)} .
$$

Set $m=-(p+2 q-2)$. For each $\beta \in \partial \Omega$, let $\Omega_{\beta}(R)=\left\{\alpha \in \partial \Omega: \mathbf{p}\left(\beta^{-1} \alpha\right) \leq R\right\}, R \in(0,1)$. We have

$$
\left|\int_{\Omega_{\beta}(R)} \phi(\alpha) \Phi(\alpha, \beta) d S(\alpha)\right| \leq 2 c\|\phi\|_{\infty} \int_{\Omega_{\beta}(R)} \mathbf{p}\left(\beta^{-1} \alpha\right)^{m} d S(\alpha) .
$$

It can be observed from [16] that $d S=\frac{4|X| \cdot|\mathbf{p}(\alpha)|^{2}}{\left(|X|^{4}+64\right)^{\frac{1}{2}}} d s$, where $d s$ is the Euclidean surface element. As $\Omega_{\beta}(R)$ is bounded, we have

$$
\left|\int_{\Omega_{\beta}(R)} \phi(\alpha) \Phi(\alpha, \beta) d S(\alpha)\right| \leq c_{1}\|\phi\|_{\infty} \int_{\Omega_{\beta}(R)} \mathbf{p}\left(\beta^{-1} \alpha\right)^{m} d s(\alpha)
$$

for some positive constant $c_{1}$. Let $\mathbf{n}$ denote the unit inward normal to the surface $\partial \Omega$ at the point $\beta$. We denote by $\Pi$ the map $\Omega_{\beta}(R) \ni \alpha \mapsto \alpha-\langle\alpha, \mathbf{n}\rangle \mathbf{n}$. Clearly, $\Pi$ is a projection of the set $\Omega_{\beta}(R)$ onto the tangent space $T_{\beta}$ to $\partial \Omega$ at the point $\beta$. The range of $\Pi$ lies inside $\mathcal{P}_{\beta}:=\Omega_{\beta}(R) \cap T_{\beta}$ and for sufficiently small $R$, the map $\Pi$ is a bijection on its range. Moreover, $R$ may be chosen small enough so that the surface element $d s$ satisfies

$$
d s(\alpha) \leq M \cdot d s(\Pi(\alpha))
$$

for some positive constant $M$. Let $\mathcal{P}_{e}$ denote the set $\left\{\beta \alpha: \alpha \in \mathcal{P}_{\beta}\right\}$. Then $\mathcal{P}_{e}=\Omega_{e}(R) \cap L$ where $L$ is the hyperplane $\mathbf{n} \cdot g=0$ in G. By translation invariance of the surface measure $d s$ induced by the Lebesgue measure, we have

$$
\int_{\mathcal{P}_{\beta}} \mathbf{p}\left(\beta^{-1} \alpha\right)^{m} d s=\int_{\mathcal{P}_{e}} \mathbf{p}(\alpha)^{m} d s
$$

The group $O(p) \times O(q)$ acts on $\mathbf{G}$ via isometries given in [5] as $\left(O_{1}, O_{2}\right)(X, Z)=\left(O_{1} X, O_{2} Z\right)$. As the Euclidean surface measure is invariant under orthogonal maps, we may choose 
suitable $\left(O_{1}, O_{2}\right)$ so that the substitution $(X, Z)=\left(O_{1} \tilde{X}, O_{2} \tilde{Z}\right)$ gives

$$
\int_{\mathcal{P}_{e}} \mathbf{p}((X, Z))^{m} d s(X, Z)=\int_{\Omega_{e}(R) \cap L_{0}} \mathbf{p}((\tilde{X}, \tilde{Z}))^{m} d s(\tilde{X}, \tilde{Z})
$$

where the equation of the hyperplane $L_{0}$ is $x_{1}=0$ when in standard local coordinates, $X$ is represented as $\left(x_{1}, x_{2}, \ldots, x_{p}\right)$. From [1, Theorem 5.12], we parameterize $\Omega_{e}(R) \cap L_{0}$ using the polar coordinates $(a, \varphi, \tilde{x}, \tilde{z}) \in(0, R) \times\left(0, \frac{\pi}{2}\right] \times S^{p-2} \times S^{q-1}$ in the following manner:

$$
\tilde{X}=a \sqrt{\cos \varphi} \tilde{x}, \quad \tilde{Z}=\frac{a^{2}}{4} \sin \varphi \tilde{z},
$$

where $\tilde{x}=\frac{1}{\|\tilde{X}\|}\left(\tilde{x}_{2}, \tilde{x}_{3}, \ldots, \tilde{x}_{p}\right), \tilde{z}=\frac{\tilde{Z}}{\|\tilde{Z}\|}$, and the measure $d s$ is given by

$$
d s=\frac{1}{4^{q}} a^{p+2 q-1} \cos ^{\frac{p}{2}-1} \varphi \sin ^{q-1} \varphi d a d \varphi d \tilde{x} d \tilde{z},
$$

where $d \tilde{x}$ and $d \tilde{z}$ denote the usual surface area measures on $S^{p-2}$ and $S^{q-1}$, respectively. By the substitution relations, we have $a=\mathbf{p}(\tilde{X}, \tilde{Z})=\mathbf{p}(X, Z)$. If $(\tilde{X}, \tilde{Z})$ is a point that corresponds to $\Pi(\alpha)$ for $\alpha \in \Omega_{\beta}(R)$ then $a \leq \mathbf{p}(\alpha)$ so that $a^{m} \geq \mathbf{p}(\alpha)^{m}$ as $m<0$. Using [1, Eq. (1.1)], we have

$$
\int_{\mathcal{P}_{\beta}} \mathbf{p}(\alpha)^{m} d s \leq \frac{1}{4^{q}} \int_{0}^{R} a d a \int_{0}^{\frac{\pi}{2}} \cos ^{\frac{p}{2}-1} \varphi \sin ^{q-1} \varphi d \varphi \int_{S^{p-2}} d \tilde{x} \int_{S^{q-1}} d \tilde{z} .
$$

Hence, the integral exists on $\Omega_{\beta}(R)$. Moreover, we have

$$
\begin{aligned}
\left|\int_{\partial \Omega \backslash \Omega_{\beta}(R)} \phi(\alpha) \Phi(\alpha, \beta) d S(\alpha)\right| & \leq 2 c\|\phi\|_{\infty} \int_{\partial \Omega \backslash \Omega_{\beta}(R)} R^{-(p+2 q-2)} d S(\alpha), \\
& \leq \frac{2 c\|\phi\|_{\infty}}{R^{(p+2 q-2)}}|\partial \Omega| .
\end{aligned}
$$

Hence, the integral exists for all $\beta \in \partial \Omega$. Further, since $d S$ is a Radon measure, a routine proof may be given to establish the uniform continuity of convolution of two integrable functions with respect to $d S$ over a compact set. In particular, $V$ is continuous.

For fixed $\beta \in \partial \Omega$, define

$$
\tilde{V}_{ \pm}(\beta)=\lim _{h \rightarrow 0+} \tilde{V}(\beta \pm h \hat{\beta}) \quad \text { and } \quad \partial^{\perp} V_{ \pm}(\beta)=\lim _{h \rightarrow 0+} \hat{\beta} \cdot \nabla V(\beta \pm h \hat{\beta})
$$

where $\hat{\beta}$ denotes the unit normal in the direction of $\beta$ and limit is in the sense of uniform convergence over the compact neighborhoods of $\beta$. In what follows, we show that the limits exist and hence determine their values.

Lemma 4.6 The kernel $\Phi$ satisfies

$$
\left|\partial^{\perp} \Phi(\alpha, \beta)\right| \leq C \mathbf{p}\left(\beta^{-1} \alpha\right)^{-(p+2 q+2)}
$$

whenever $\beta \neq \alpha$ and for some $C>0$, depending on $\alpha$. 
Proof We have

$$
\Phi(\alpha, \beta)=\Phi_{e}\left(\beta^{-1} \alpha\right)=2 c \mathbf{p}\left(\beta^{-1} \alpha\right)^{-(p+2 q-2)} .
$$

It can be observed from [16, Eq. (1.12)] that

$$
\partial^{\perp}=\left({\frac{|X|^{6}}{16}}^{6}+|Z|^{2}|X|^{2}\right)^{-1}\left({\frac{|X|^{4}}{4}}^{4}+J_{Z}\right) X \quad \text { for all }(X, Z) \in \partial \Omega \text { such that }|X| \neq 0 \text {. }
$$

For $\alpha=(X, Z)$ and $\beta=\left(X^{\prime}, Z^{\prime}\right)$ in $\mathbf{G}$ for which $|X| \neq 0$, we write

$$
\begin{aligned}
\frac{\partial}{\partial n_{0}} \Phi(\alpha, \beta)= & -\frac{c}{2}(p+2 q-2)\left(\mathbf{p}\left(\beta^{-1} \alpha\right)\right)^{-(p+2 q+2)}\left(|X|^{6}+16|Z|^{2}|X|^{2}\right)^{-1}\left\{|X|^{4}\right. \\
& \times\left(|X|^{2}+\left|X^{\prime}\right|^{2}\right)+2|X|^{2}\left|X^{\prime}\right|\left|P_{\left(\mathbb{R}+J_{\mathfrak{z}}\right) X^{\prime}} X\right|\left(X^{\prime} \cdot X+\sum_{j=1}^{q} J_{Z j} X^{\prime} \cdot X\right) \\
& -2|X|^{4}\left\langle X^{\prime}, X\right\rangle-|X|^{2}\left(|X|^{2}+\left|X^{\prime}\right|^{2}\right) X^{\prime} \cdot X+4|X|^{2} J_{Z^{\prime}-Z} X^{\prime} \cdot X \\
& +8\left|X^{\prime}\right|\left|P_{\left(\mathbb{R}+J_{\mathfrak{z}}\right) X^{\prime}} X\right|\left(X^{\prime} \cdot J_{z} X+\sum_{j=1}^{q} J_{Z_{j}} X^{\prime} \cdot J_{z} X\right)-4\left(|X|^{2}+\left|X^{\prime}\right|^{2}\right) \\
& \left.\times X^{\prime} \cdot J_{Z} X+16 J_{Z^{\prime}} X^{\prime} \cdot J_{Z} X-16 J_{Z} X^{\prime} \cdot J_{Z} X\right\}
\end{aligned}
$$

where "." represents the Euclidean dot-product. Clearly,

$$
\lim _{|X| \rightarrow 0} \frac{\partial}{\partial n_{0}} \Phi(\alpha, \beta)=0 .
$$

Hence $\Phi(\cdot, \beta) \in \mathfrak{C}(\Omega)$. Now for $\beta \in \partial \Omega$ there exists $\epsilon>0$ such that $|\sigma(\alpha)|>\frac{|\sigma(\beta)|}{2}$ for every $\alpha \in B_{\epsilon}(\beta)$ where $B_{\epsilon}(\beta)=\left\{\alpha: \mathbf{p}\left(\alpha^{-1} \beta\right)<\epsilon\right\}$ and $\sigma(\alpha)=\sigma(X, Z)={\frac{|X|^{6}}{16}}^{6}+|Z|^{2}\left|X^{2}\right|$. Since $\Omega$ is bounded, for $\beta \neq \alpha$

$$
\left|\partial^{\perp} \Phi(\alpha, \beta)\right| \leq c^{\prime}\left(\frac{p+2 q-2}{4}\right) \mathbf{p}\left(\beta^{-1} \alpha\right)^{-(p+2 q+2)}
$$

for some $c^{\prime}>0$.

Lemma 4.7 On the boundary $\partial \Omega$, the kernel $\Phi$ satisfies

$$
\int_{\partial \Omega} \partial^{\perp} \Phi(\alpha, \beta) d S(\alpha)= \begin{cases}-2, & \beta \in \Omega, \\ -1, & \beta \in \partial \Omega, \\ 0, & \beta \in \mathbf{G} \backslash \bar{\Omega} .\end{cases}
$$

Proof Using appropriate substitutions in (3.1), the value of the integral on $\Omega$ and $\mathbf{G} \backslash \bar{\Omega}$ is obvious. For $\beta \in \partial \Omega$ and $r>0$, set $\Omega_{\beta}(r)=\left\{\alpha \in \mathbf{G}: \mathbf{p}\left(\beta^{-1} \alpha\right)<r\right\}$. Now using Green's formula (3.1) with $u=1$ and $v=\Phi(\cdot, \beta)$ on $\Omega \backslash \Omega_{\beta}(r)$, we have

$$
\int_{\partial\left(\Omega \backslash \Omega_{\beta}(r)\right)} \partial^{\perp} \Phi(\alpha, \beta) d S(\alpha)=0,
$$


which implies

$$
\begin{aligned}
\lim _{r \rightarrow 0} \int_{\partial \Omega \backslash \Omega_{\beta}(r)} \partial^{\perp} \Phi(\alpha, \beta) d S(\alpha) & =-\lim _{r \rightarrow 0} \int_{\Omega \cap \partial \Omega_{\beta}(r)} \partial^{\perp} \Phi(\alpha, \beta) d S(\alpha) \\
& =-\frac{1}{2} \lim _{r \rightarrow 0} \int_{\partial \Omega_{\beta}(r)} \partial^{\perp} \Phi(\alpha, \beta) d S(\alpha) .
\end{aligned}
$$

From [16, Eq. (1.15)] and (2.3), we finally have

$$
\int_{\partial \Omega} \partial^{\perp} \Phi(\alpha, \beta) d S(\alpha)=-1
$$

Corollary 4.8 For $\phi \in C(\partial \Omega)$ and $\beta \in \partial \Omega$,

$$
\int_{\partial \Omega} \phi(\alpha) \partial^{\perp} \Phi(\alpha, \beta) d S(\alpha)<\infty
$$

Lemma 4.9 For sufficiently small $h_{0}>0$, consider a small neighborhood $N_{\partial \Omega}\left(h_{0}\right)$ of $\partial \Omega$ that consists of points $\beta \in \mathbf{G}$ which can be represented uniquely in the form $\beta=\gamma+h \hat{\gamma}$, where $\gamma \in \partial \Omega$ and $h \in\left[-h_{0}, h_{0}\right]$. Define

$$
u(\beta)=\int_{\partial \Omega}\{\phi(\alpha)-\phi(\gamma)\} \partial^{\perp} \Phi(\alpha, \beta) d S(\alpha)
$$

for $\beta \in N_{\partial \Omega}\left(h_{0}\right) \backslash \partial \Omega$. Then

$$
\lim _{h \rightarrow 0+} u(\gamma+h \hat{\gamma})=u(\gamma), \quad \gamma \in \partial \Omega
$$

uniformly over compact neighborhoods in $N_{\partial \Omega}\left(h_{0}\right)$.

Proof Define $\partial \Omega(\gamma ; r)=\partial \Omega \cap \Omega[\gamma ; r]$ where $\Omega[\gamma ; r]=\left\{\alpha \in \mathbf{G}: \mathbf{p}\left(\alpha^{-1} \gamma\right) \leq r\right\}$ and let $\mathbf{p}\left(\beta^{-1} \gamma\right)=\lambda$. Take $r<\mathbf{p}\left(\beta^{-1} \gamma\right)$ for a sufficiently small $r>0$. Using $\mathbf{p}\left(\beta^{-1} \gamma\right) \leq \mathbf{p}\left(\beta^{-1} \alpha\right)+$ $\mathbf{p}\left(\alpha^{-1} \gamma\right)$, we have $\lambda-r \leq \mathbf{p}\left(\beta^{-1} \alpha\right)$.

Consider

$$
\begin{aligned}
\int_{\partial \Omega(\gamma ; r)} \partial^{\perp} \Phi(\alpha, \beta) d S(\alpha) & \leq c_{1} \int_{\partial \Omega(\gamma ; r)} \mathbf{p}\left(\beta^{-1} \alpha\right)^{-(p+2 q+2)} d S(\alpha), \beta \neq \alpha \\
& \leq c_{1} \int_{\partial \Omega(\gamma ; r)} \frac{1}{(\lambda-r)^{(p+2 q+2)}} d S(\alpha) \\
& \leq c_{1} \frac{1}{(\lambda-1)^{(p+2 q+2)}}|\partial \Omega(\gamma ; r)|,
\end{aligned}
$$

where $p, q$ denote the dimension of $\mathfrak{v}$ and $\mathfrak{z}$, respectively. The mean value theorem implies

$$
\begin{aligned}
\left|\partial^{\perp} \Phi(\alpha, \beta)-\partial^{\perp} \Phi(\alpha, \gamma)\right| & \leq c_{2} \mathbf{p}\left(\beta \gamma^{-1}\right)\left|\left(\nabla_{\gamma}\right)\left(\partial^{\perp} g_{\beta}(\alpha)\right)\right| \\
& \leq c_{3} \frac{\mathbf{p}\left(\beta \gamma^{-1}\right)}{\mathbf{p}\left(\gamma \alpha^{-1}\right)^{(p+2 q+2)}} .
\end{aligned}
$$


Hence,

$$
\int_{\partial \Omega \backslash \partial \Omega(\gamma ; r)}\left|\partial^{\perp} \Phi(\alpha, \beta)-\partial^{\perp} \Phi(\alpha, \gamma)\right| d S(\alpha) \leq c_{4} \frac{\mathbf{p}\left(\beta \gamma^{-1}\right)}{\mathbf{p}\left(\gamma \alpha^{-1}\right)^{(p+2 q+2)}},
$$

for $c_{4}>0$. Combining (4.7) and (4.8), we obtain

$$
|u(\beta)-u(\gamma)| \leq c\left\{\max _{\alpha \in \Omega[\gamma ; r]}|\phi(\alpha)-\phi(\gamma)|+\frac{\mathbf{p}\left(\beta \gamma^{-1}\right)}{r^{(p+2 q+2)}}\right\}
$$

for $r$ sufficiently small and a positive constant $c$.

As $\phi$ is uniformly continuous on $\partial \Omega$, for any given $\epsilon>0$, there exists $\delta>0$ such that

$$
\max _{\alpha \in \Omega[\gamma ; r]}|\phi(\alpha)-\phi(\gamma)| \leq \frac{\epsilon}{2 c}
$$

for all $\gamma \in \partial \Omega$. Taking $\delta<\frac{\epsilon r^{p+2 q+2}}{2 c}$, we get $|u(\beta)-u(\gamma)|<\epsilon$ for all $\mathbf{p}\left(\beta \gamma^{-1}\right)<\delta$.

Theorem 4.10 Let $\phi \in C(\partial \Omega)$. Then it is possible to extend $\tilde{V}$ from $\Omega$ to $\bar{\Omega}$ and from $\mathbf{G} \backslash \bar{\Omega}$ to $\mathbf{G} \backslash \Omega$ in a continuous fashion with the following limiting values:

$$
\tilde{V}_{ \pm}(\beta)=\int_{\partial \Omega} \phi(\alpha) \partial^{\perp} \Phi(\alpha, \beta) d S(\alpha) \pm \phi(\beta), \quad \beta \in \partial \Omega
$$

Proof By Corollary 4.8, the above integral is a continuous function on $\partial \Omega$. Take $N_{\partial \Omega}\left(h_{0}\right)$ as in the previous lemma and write $\tilde{V}$ in the following form:

$$
\tilde{V}(\beta)=u(\beta)+\phi(\gamma) \omega(\beta), \quad \beta=\gamma+h \hat{\gamma} \in N_{\partial \Omega}\left(h_{0}\right) \backslash \partial \Omega,
$$

where

$$
u(\beta)=\int_{\partial \Omega}\{\phi(\alpha)-\phi(\gamma)\} \partial^{\perp} \Phi(\alpha, \beta) d S(\alpha)
$$

and

$$
\omega(\beta)=\int_{\partial \Omega} \partial^{\perp} \Phi(\alpha, \beta) d S(\alpha) .
$$

Now using Lemma 4.9, the proof follows.

Theorem 4.11 For $\phi \in C(\partial \Omega)$, we have

$$
\partial^{\perp} V_{ \pm}(\beta)=\int_{\partial \Omega} \phi(\alpha) \partial^{\perp} \Phi(\alpha, \beta) d S(\alpha) \pm \phi(\beta), \quad \beta \in \partial \Omega .
$$

Proof Let $\tilde{V}$ and $N_{\partial \Omega}\left(h_{0}\right)$ be as in the proof of Theorem 4.10. Then for $\beta=\gamma+h \hat{\gamma} \in$ $N_{\partial \Omega}\left(h_{0}\right) \backslash \partial \Omega$ and using $(\nabla)_{\beta}\left(\Phi(\alpha, \beta)=(\nabla)_{\alpha}(\Phi(\alpha, \beta)\right.$, we can write

$$
\hat{\gamma} \cdot \nabla(V(\beta))+\tilde{V}(\beta)=\int_{\partial \Omega}\{\hat{\gamma}+\hat{\beta}\} \cdot \nabla_{\alpha}(\Phi(\alpha, \beta) \phi(\alpha) d S(\alpha) .
$$


Analogous to $\tilde{V}$ in Theorem 4.10, the right-hand side can be shown to be continuous on $N_{\partial \Omega}\left(h_{0}\right)$. Now, the remaining proof follows from Theorem 4.10.

Theorem 4.12 The following limit holds uniformly for all $\beta \in \partial \Omega$ :

$$
\lim _{h \rightarrow 0+} \hat{\beta} \cdot\{\nabla \tilde{V}(\beta+h \hat{\beta})-\nabla \tilde{V}(\beta-h \hat{\beta})\}=0
$$

Proof The proof follows along similar lines to the proof of Theorem 4.10.

\section{Existence of solution}

Define integral operators $W_{1}, W_{2}: C(\partial \Omega) \rightarrow C(\partial \Omega)$ as

$$
\left(W_{1} \phi\right)(\beta):=\int_{\partial \Omega} \phi(\alpha)\left(\partial^{\perp} \Phi(\alpha, \beta)\right)_{\alpha} d S(\alpha), \quad \beta \in \partial \Omega
$$

and

$$
\left(W_{2} \psi\right)(\beta):=\int_{\partial \Omega} \psi(\alpha)\left(\partial^{\perp} \Phi(\alpha, \beta)\right)_{\beta} d S(\alpha), \quad \beta \in \partial \Omega .
$$

As $\partial \Omega$ is of class $C^{1}$ and by using the estimate (4.5), we can easily conclude that $W_{1}, W_{2}$ are compact. Also with respect to the dual system $\langle C(\partial \Omega), C(\partial \Omega)\rangle$, defined by

$$
\langle\phi, \psi\rangle:=\int_{\partial \Omega} \phi \psi d S \quad \phi, \psi \in C(\partial \Omega)
$$

the operators $W_{1}$ and $W_{2}$ are adjoint.

Theorem 5.1 The operators $I+W_{1}, I+W_{2}$ have one-dimensional nullspaces.

Proof Let $N\left(I+W_{1}\right)$ denote the nullspace of $I+W_{1}, \phi \in N\left(I+W_{1}\right)$ and let $\tilde{V}$ be the corresponding double layer potential. By Theorem 4.10, $\tilde{V}$ is bounded and harmonic in $\mathbf{G} \backslash \Omega$. By (4.9), $\tilde{V}_{+}=\phi+W_{1} \phi=0$ on $\partial \Omega$ and therefore $\tilde{V}$ can be extended continuously on $\Omega$ by zero. Let $\tilde{V}^{\prime}$ denote this extension. Then $\tilde{V}^{\prime}$ is bounded harmonic on $\mathbf{G}$ and by Liouville theorem for the sub-Laplacian [3, Theorem 5.8.7], $\tilde{V}^{\prime}=0$ on $\mathbf{G} \backslash \bar{\Omega}$. Hence using [3, Theorem 8.2.16], $\tilde{V}=0$ a.e. on $\mathbf{G} \backslash \bar{\Omega}$. Moreover, from Theorem 4.12, $\partial^{\perp} \tilde{V}_{-}=0$ on $\partial \Omega$. Uniqueness of the exterior Neumann problem then implies that $\phi$ is constant on $\Omega$. Again from (4.9), we deduce that $\phi$ is constant on $\partial \Omega$. So $N\left(I+W_{1}\right) \subset \operatorname{span}\{s\}$, where $s$ is a constant. Using Lemma 4.7, we have $s+K s=0$. and hence $N\left(I+W_{1}\right)=\operatorname{span}\{s\}$. By first Fredholm theorem [17], $N\left(I+W_{2}\right)$ also has dimension one.

Theorem 5.2 For $\psi \in C(\partial \Omega)$, the single layer potential

$$
V(\beta):=\int_{\partial \Omega} \psi(\alpha) \Phi(\alpha, \beta) d S(\alpha), \quad \beta \in \Omega
$$

is a solution of the interior Neumann problem provided $\psi$ solves the following integral equation:

$$
\psi(\beta)+\int_{\partial \Omega} \psi(\alpha) \partial^{\perp} \Phi(\alpha, \beta) d S(\alpha)=g(\beta), \quad \beta \in \partial \Omega .
$$


Proof The proof follows from Theorem 4.11.

Theorem 5.3 The interior Neumann problem is solvable if and only if

$$
\int_{\partial \Omega} g d S=0
$$

Proof (Necessity) This can be proved using the identity (3.1). For $v=1$ and a solution $u$ of the interior Neumann problem (3.2), we have

$$
\int_{\partial \Omega} g d S=0
$$

(Sufficiency) By Fredholm's theorem, the inhomogeneous problem $\psi+W_{2} \psi=g$ has a solution if and only if $g$ is orthogonal to a solution of $\phi+W_{1} \phi=0$. By Theorem 5.1, it is equivalent to $\langle 1, g\rangle=0$, i.e.,

$$
\int_{\partial \Omega} g d S=0
$$

Now using Theorem 5.2, the interior Neumann problem has a solution.

\section{Acknowledgements}

The authors are grateful to the reviewers for their constructive comments and suggestions, which have greatly improved this paper.

\section{Funding}

Research of the second author is supported by the Council of Scientific \& Industrial Research (CSIR), Government of India (Ref. No.: 09/045(1479)/2017-EMR-I).

Availability of data and materials

Not applicable.

Competing interests

The authors declare that no competing interests exist.

\section{Authors' contributions}

The authors contributed equally to this paper. All authors read and approved the final manuscript.

\section{Author details}

${ }^{1}$ Department of Mathematics, Hansraj College, University of Delhi, Delhi, India. ${ }^{2}$ Department of Mathematics, Faculty of Mathematical Sciences, University of Delhi, Delhi, India.

\section{Publisher's Note}

Springer Nature remains neutral with regard to jurisdictional claims in published maps and institutional affiliations.

Received: 19 March 2020 Accepted: 30 April 2020 Published online: 13 May 2020

\section{References}

1. Balogh, Z.M., Tyson, J.T.: Polar coordinates in Carnot groups. Math. Z. 241(4), 697-730 (2002)

2. Beals, R., Gaveau, B., Greiner, P.: The Green function of model step two hypoelliptic operators and the analysis of certain tangential Cauchy-Riemann complexes. Adv. Math. 121(2), 288-345 (1996)

3. Bonfiglioli, A., Lanconelli, E., Uguzzoni, F.: Stratified Lie Groups and Potential Theory for Their Sub-Laplacians. Springer Monographs in Mathematics. Springer, Berlin (2007)

4. Bramanti, M.: An Invitation to Hypoelliptic Operators and Hörmander's Vector Fields. SpringerBriefs in Mathematics. Springer, Cham (2014)

5. Cowling, M., Dooley, A.H., Korányi, A., Ricci, F.: H-Type groups and Iwasawa decompositions. Adv. Math. 87(1), 1-41 (1991)

6. Dubey, S., Kumar, A., Mishra, M.M.: The Neumann problem for the Kohn-Laplacian on the Heisenberg group $\mathbb{H}_{n}$. Potential Anal. 45(1), 119-133 (2016) 
7. Evans, L.C.: Partial Differential Equations, 2nd edn. Graduate Studies in Mathematics, vol. 19. Am. Math. Soc. Providence (2010)

8. Franchi, B., Serapioni, R., Serra Cassano, F.: On the structure of finite perimeter sets in step 2 Carnot groups. J. Geom. Anal. 13(3), 421-466 (2003)

9. Gaveau, B.: Principe de moindre action, propagation de la chaleur et estimées sous elliptiques sur certains groupes nilpotents. Acta Math. 139(1-2), 95-153 (1977)

10. Gilbarg, D., Trudinger, N.S.: Elliptic Partial Differential Equations of Second Order, vol. 224, 2nd edn. Springer, Berlin (1983)

11. Jerison, D.S.: The Dirichlet problem for the Kohn Laplacian on the Heisenberg group. I. J. Funct. Anal. 43(1), 97-142 (1981)

12. Jerison, D.S.: The Dirichlet problem for the Kohn Laplacian on the Heisenberg group. II. J. Funct. Anal. 43(2), 224-257 (1981)

13. Kaplan, A.: Fundamental solutions for a class of hypoelliptic PDE generated by composition of quadratic forms. Trans. Am. Math. Soc. 258(1), 147-153 (1980)

14. Kellogg, O.D.: Foundations of Potential Theory. Die Grundlehren der Mathematischen Wissenschaften, vol. 31 Springer, Berlin (1929). Reprint from the first edition of, 1967

15. Korányi, A.: Geometric properties of Heisenberg-type groups. Adv. Math. 56(1), 28-38 (1985)

16. Korányi, A.: Poisson formulas for circular functions on some groups of type H. Sci. China Ser. A 49(11), 1683-1695 (2006)

17. Kress, R.: Linear Integral Equations. Applied Mathematical Sciences., vol. 82. Springer, Berlin (1989)

18. Liu, Z.: Existence results for the general Schrödinger equations with a superlinear Neumann boundary value problem. Bound. Value Probl. 2019,61 (2019)

19. Polidoro, S., Ragusa, M.A.: Harnack inequality for hypoelliptic ultraparabolic equations with a singular lower order term. Rev. Mat. Iberoam. 24(3), 1011-1046 (2008)

\section{Submit your manuscript to a SpringerOpen ${ }^{\circ}$} journal and benefit from:

- Convenient online submission

- Rigorous peer review

- Open access: articles freely available online

- High visibility within the field

- Retaining the copyright to your article

Submit your next manuscript at $\boldsymbol{\nabla}$ springeropen.com 